\title{
Postpartum pneumomediastinum and subcutaneous emphysema (Hamman's Syndrome)
}

\author{
Awatuf Elshirif* and Jaya Tyagi-Bhatia \\ Department of Obstetrics and Gynaecology, Kings College Hospital NHS Foundation Trust, London, UK
}

\section{Introduction}

Hamman (1939) first described postpartum pneumomediastinum in association with subcutaneous emphysema. This condition, also referred to as Hamman's syndrome, is rare, with only about 200 cases reported worldwide [1], with an estimated incidence of 1 in 100,000 vaginal deliveries [2]. Despite its daunting presentation, the condition is usually minimal and the course is self-limiting [1]. Subcutaneous emphysema in the neck has also been linked to vomiting during pregnancy [3]. Chest pain is the most common symptom of pneumomediastinum although the severity depends on the location and amount of air (Hamman 1939). Additional symptoms may include dyspnoea, cough, and palpitations. A definitive diagnosis is made radiographically [1].

\section{Case report}

A 27 year old Caucasian primigravida lady was admitted at 41 weeks of gestation to our birthing unit in early labour. She was non smoker, with a BMI (Body Mass Index) of $29 \mathrm{~kg} / \mathrm{m}^{2}$. She had no past medical or surgical history of note and had an uneventful antenatal course.

Sixteen hours after onset of labour, she progressed to full dilation, with vertex presentation, in the right occiput-posterior position. Her second stage of labour was prolonged to 3 hours. She used Entonox for pain control and had a spontaneous vaginal delivery of a healthy male baby weighing $4570 \mathrm{~g}$ with an episiotomy. The third stage of labour lasted five minutes and the episiotomy was subsequently repaired. The immediate postpartum period was unremarkable and she was clinically stable with a blood pressure $126 / 80 \mathrm{~mm}$ of $\mathrm{Hg}$ and pulse of 88 /minute.

However, four hours following the delivery, she complained of chest heaviness, tightness and a swollen neck. On examination, her blood pressure was $110 / 60 \mathrm{mmHg}$, pulse was $80 /$ minute, respiratory rate was $16 /$ minute and Oxygen saturation was $97 \%$ on the air. On auscultation, chest was clear with bilateral air entry with equal expansion. Palpation of neck and chest revealed subcutaneous crepitation and a diagnosis of subcutaneous emphysema was established. The chest X-ray was ordered which showed pneumomediastinum and subcutaneous emphysema of the neck but no pneumothorax (Figure 1a).

Double contrast CT of the neck and chest (Figure $1 \mathrm{~b}$ ) revealed significant pneumomediastinum with possible interruption of the right posterolateral aspect of the trachea at the level of the second vertebral body with possibility of a tracheal rupture. It also showed substantial subcutaneous emphysema of the anterior chest wall extending to the root of the neck and extending superiorly to the level of the skull base. There was no evidence of pneumothorax.
She was monitored closely with conservative management (bed rest, analgesia, oxygen and antibiotics), which led to a gradual resolution of subcutaneous emphysema and pneumomediastinum.

She was discharged on day 4 after delivery and a repeat chest $\mathrm{X}$ ray on day 7 showed complete resolution.

\section{Discussion}

Hamman's syndrome is a rare complication of labour and delivery. It usually occurs in the second stage of labour; nevertheless, it is often clinically apparent in postpartum phase.

The prolonged Valsalva manoeuvre (straining with the glottis closed) during the second stage of labour as well as the screaming leads to a rupture of marginally situated alveoli into the pulmonary interstitial space, with tracking of air along the bronchovascular connective tissue planes towards the hilum and mediastinum [4]. Subcutaneous emphysema results when air escapes from the mediastinum into the subcutaneous and deep tissues of the neck. When subcutaneous emphysema in the neck and pneumothorax coexist it is likely that both are secondary to mediastinal emphysema, with perforation of the mediastinal pleura resulting in the pneumothorax [4].

According to Macklin and Macklin [5] common presenting symptoms of pneumomediastinum include retrosternal chest pain, dyspnoea, pleurisy, facial and neck pain, odynophagia, or dysphagia (which can be secondary to pressurized air from alveolar rupture tracking between tissue planes). Chest pain in labour and pregnancy may be due to a number of causes. Since pneumomediastinum is extremely rare, it is essential to exclude pulmonary embolism, amniotic-fluid embolism, myocardial infarction, pneumothorax, and aortic dissection before making this unusual diagnosis.

A thorough history and clinical examination and chest radiograph is often enough to diagnose Spontaneous pneumomediastinum [6]. $30 \%$ of spontaneous pneumomediastinum cases may not be easily seen on routine chest X-ray. Double-contrast CT imaging may be helpful, particularly if the air-leak involves the posterior mediastinum [6,7].

Spontaneous pneumo mediastinum is often a benign and selflimiting condition. It is managed conservatively with bedrest, observation, and analgesia. Other complications such as tension

Correspondence to: Awatuf Elshirif, Department of Obstetrics and Gynaecology, King's College Hospital NHS foundation trust, Princess Royal university Hospital, Farnborough, BR6 8ND, UK; E-mail: awatuf.elshirif@nhs.net

Received: March 12, 2015; Accepted: April 01, 2015; Published: April 04, 2015 

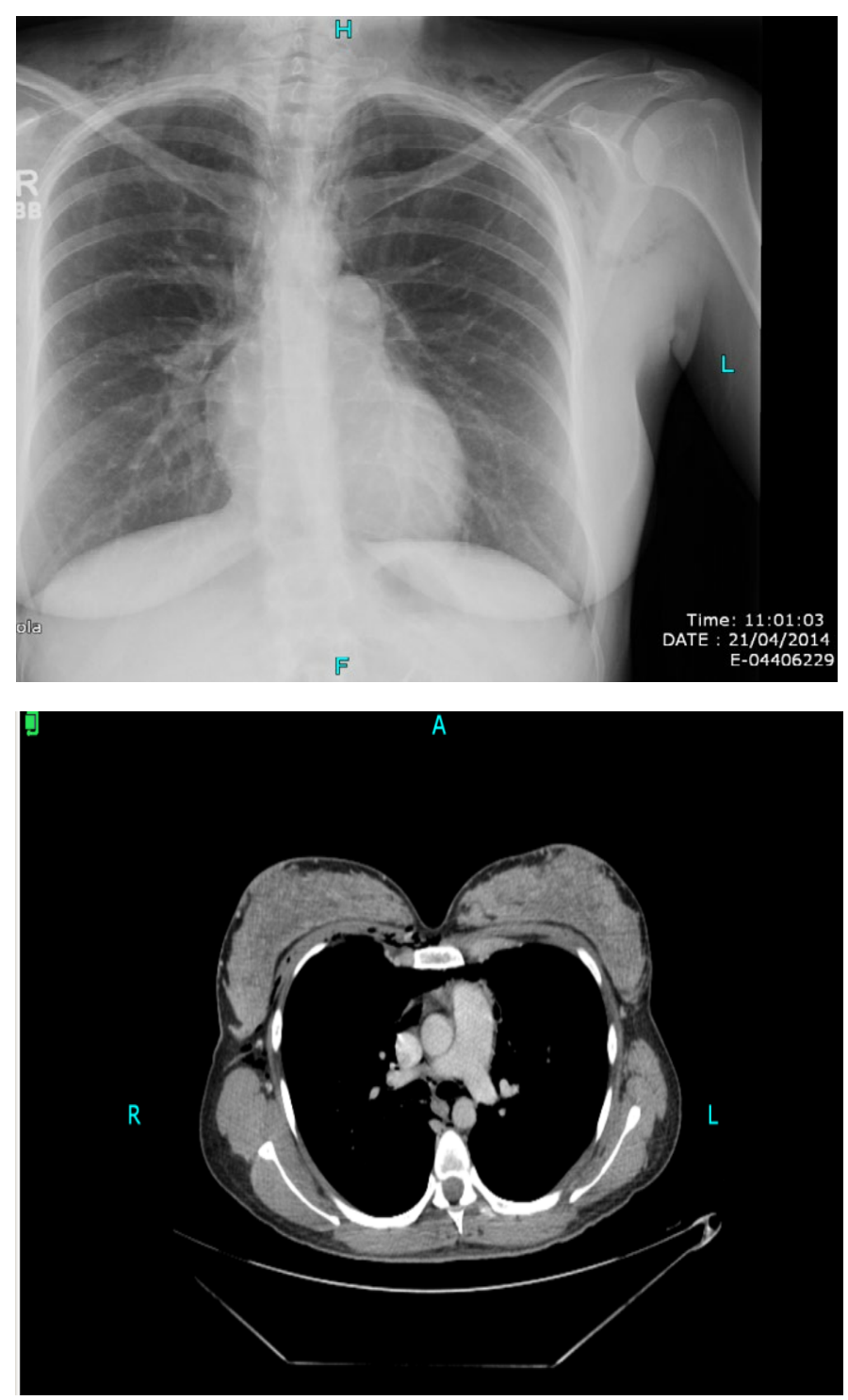

Figure 1. (a) showing pneumomediastinum and subcutaneous emphysema of the neck but no pneumothorax. (b) showing significant pneumomediastinum and substantial subcutaneous emphysema. pneumomediastinum and pneumothorax are rare. There is currently no reliable data to suggest the rate of recurrence of Spontaneous pneumomediastinum in pregnancy [7]. The use of nitrous oxide increases the risk of gas trapping and may increase the risk of pneumomediastinum [8]. In patients with a history of pneumomediastinum, it may therefore be prudent to avoid the use of nitrous oxide. While the second stage of labour is generally shorter for multiparous patients, epidural analgesia may be discussed as a method of avoiding premature or prolonged pushing in the late first or early second stages of labour. If spontaneous pneumomediastinum is recognized early, it is felt that delivery should be hastened (for example, by Ventouse, forceps, or urgent cesarean section) to minimize prolonged or strenuous valsalva and progression of mediastinal emphysema.

\section{Declaration of interest}

The authors report no conflicts of interest. The authors alone are responsible for the content and writing of the Paper.

\section{References}

1. Seidl JJ, Brotzman GL (1994) Pneumomediastinum and subcutaneous emphysem following vaginal delivery. Case report and review of the literature. J Fam Pract 39: 178-180. [Crossref]

2. Heffner JE, Sahn SA (1992) Pleural disease in pregnancy. Clin Chest Med 13: 667678. [Crossref]

3. Woolford TJ, Birzgalis AR, Lundell C, Farrington WT (1993) Vomiting in pregnancy resulting in oesophageal perforation in a 15-year-old. J Laryngol Otol 107: 1059-1060. [Crossref]

4. HammanL (1945) Mediastinal emphysema. JAMA 128: 1-6.

5. Macklin MT, Macklin CC (1944) Malignant interstitial emphysema of the lungs and mediastinum as an important occult complication in many respiratory diseases and other conditions: an interpretation of the clinical literature in light of laboratory experiment. Medicine 23: 281-358.

6. Kaneki T, Kubo K, Kawashima A, Koizumi T, Sekiguchi M, et al. (2000) Spontaneous pneumomediastinum in 33 patients: yield of chest computed tomography for the diagnosis of the mild type. Respiration 67: 408-411. [Crossref]

7. Revicky V, Simpson P, Fraser D (2010) Postpartum pneumomediastinum: an uncommon cause for chest pain. Obstet Gynecol Int 2010: 956142. [Crossref]

8. Jayran-Nejad Y (1993) Subcutaneous emphysema in labour. Anaesthesia 48: 139-140. [Crossref]

Copyright: (C2015 Elshirif A. This is an open-access article distributed under the terms of the Creative Commons Attribution License, which permits unrestricted use, distribution, and reproduction in any medium, provided the original author and source are credited. 